\title{
Evaluation of drag and added mass forces acting on vibrat- ing cantilever beams in the air
}

\author{
Artem Nuriev ${ }^{1, *}$, Airat Kamalutdinov ${ }^{1}$, Olga Zaitseva $^{2}$, and Boudkhil Affane ${ }^{1}$ \\ ${ }^{1}$ Lobachevskii Institute of Mathematics and Mechanics, Kazan Federal University, 18, Kremlyovskaya \\ St., Kazan, Tatarstan 420008, Russian Federation \\ ${ }^{2}$ Higher Institute of Information Technology and Information Systems, Kazan Federal University, 18, \\ Kremlyovskaya St., Kazan, Tatarstan 420008, Russian Federation
}

\begin{abstract}
In the paper a method for determining of the aerodynamic influence on oscillating cantilever beams based on gyroscope measurements is presented. The developed method allow to establish the global influence of the aerodynamic effects on oscillation process and the local force characteristics in each cross-section of the beam. The obtained estimates of drag and added mass forces acting on the beams show a good agreement with the known numerical and experimental data.
\end{abstract}

\section{Introduction}

The past few decades have witnessed a rising interest in the study of mechanical vibrations of the beams in viscous static fluids. The motivations for these studies stem from different practical applications covering diverse fields of knowledge such as atomic microscopy [1], sensors and actuators based on micromechanical oscillators [2], cooling devices [3], marine and offshore equipments $[4,5]$.

In the general, the problem of evaluation of aerodynamic forces and moments acting on a cantilever beams is extremely complicated, mainly because of the complexity of threedimensional gas flows caused by vibrations of the beam. But in the case when the length of the beam considerably exceeds its other geometrical dimensions, at low structural vibration modes the length of the vibrational wave is much greater than deviations of the beam, as a result it can be regarded as locally planar. That allows to use a simplified quasi-two dimensional model of interaction between a beam and a gas, according to which the aerodynamic forces acting on each cross-section of the beam can be considered as a result of the planar flow past a section. This model is widely used in many studies [3, 6-11].

Applying the above simplifying assumptions, the integral relations between the logarithmic decrement of the beam oscillations (LD) and drag forces acting on each cross section of the beam and between the relative variation in frequency (RVF) and the added mass force can be established. Such integral relations are used in this work for the experimental determination of aerodynamic forces from parameters of the beam oscillations (LD and RVF).

\footnotetext{
*e-mail: Artem.Nuriev@kpfu.ru
} 


\section{Experimental measurements}

In experiments the damped oscillations caused by the initial deflection of the free end of the console from equilibrium was measured. The scheme of the experiment is shown in Figure 1. To register the vibrations of the beam the 3-axis MEMS gyroscope sensor MPU6500 was used. The sensor provides measurements of angular velocity in the range of \pm 2000 degrees/sec with sampling rate equals to $8 \mathrm{kHz}$ and accuracy up to 0.05 degrees/sec. For data transfer from the sensor to the workstation the digital protocol I2C $400 \mathrm{KHz}$ was used.

As experimental samples duralumin plates with the following geometrical parameters were used: thickness $h=0.95 \mathrm{~mm}$, width $b=20,30 \mathrm{~mm}$ and length $L$, which was varied in the range $150-450 \mathrm{~mm}$. All samples were made from a single sheet of aluminium alloy A1-2024. Note that the use of aluminium alloy is an important part of the experimental methodology, since the mechanical damping component for this material does not depend on the amplitude of the oscillation.

MEMS sensor was mounted on the test sample in the vicinity of the free end of beam along the horizontal axis of symmetry. The sensor measures 3 angular velocities $\left(\Omega_{x}, \Omega_{y}, \Omega_{z}\right)$ with respect to the coordinate system that is rigidly connected to the beam end (Figure 1).

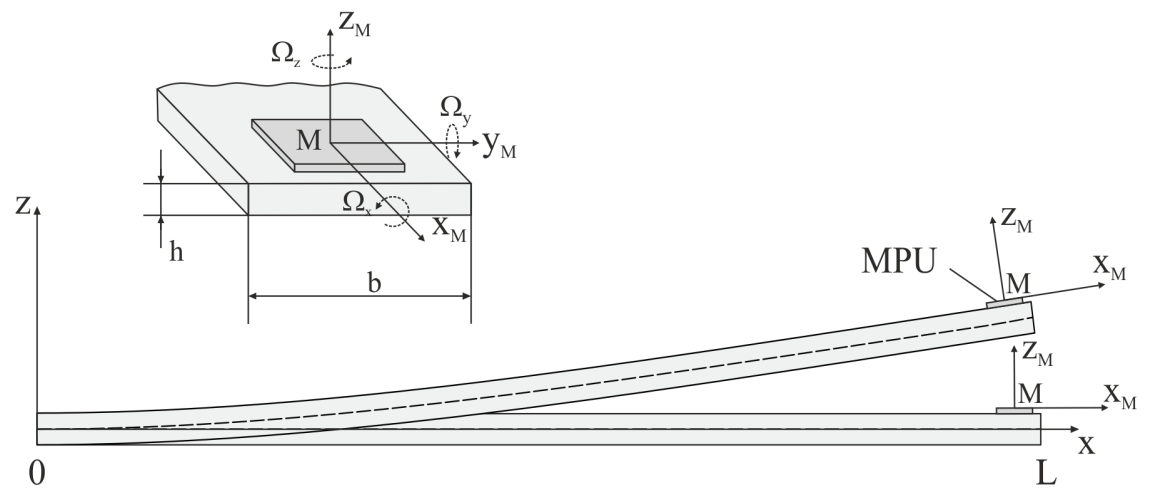

Figure 1. Scheme of MPU mounting

The geometry parameters of the test specimens, the fastening conditions and the method of excitation of oscillations prevent the occurrence of the $\Omega_{z}$ component of the angular velocity. Typical measurements of the angular velocities corresponding to the torsional and flexural component of the beam oscillations are shown in Figure 2. As can be seen, the presence of non-zero angular velocities is fixed on both axes of the gyroscope.

The fundamental flexural mode of beam oscillations is absolutely dominant in the amplitude spectrum of the signal $\Omega_{y}$. In addition to it, there is another harmonic in the spectrum. This harmonic corresponds to the second natural frequency of flexural vibrations. Its appearance is related to the conditions of the initial deflection of the beam. The amplitude of the additional harmonic in the limiting case does not exceed $10 \%$ of the amplitude of the fundamental harmonic. This ratio is rapidly decreased over time due to the high damping rate of the additional component. When we make the transition from $\Omega_{y}$ to the displacement, the contribution of the additional harmonic will decrease as the ratio of the fundamental frequency to the additional frequency (see Sect. 3 ), for this reason it can be ignored in calculations of the LD of the beam oscillations. 
The nonzero values of the other component of the angular velocity $\Omega_{x}$, which was registered in the zone of large amplitudes of the deviation of the beam from the equilibrium state, indicates the presence of the torsional oscillations of the beam. However, in all measurements the amplitude of the angular velocity of the torsional oscillations was more than two orders of magnitude smaller than the angular velocity of flexural vibrations. As a consequence, the maximum amplitude of the rotation angle of the beam in all the experiments did not exceed a hundredth part of degree. For this reason, when processing experimental data, the torsion effect cannot be taken into account.
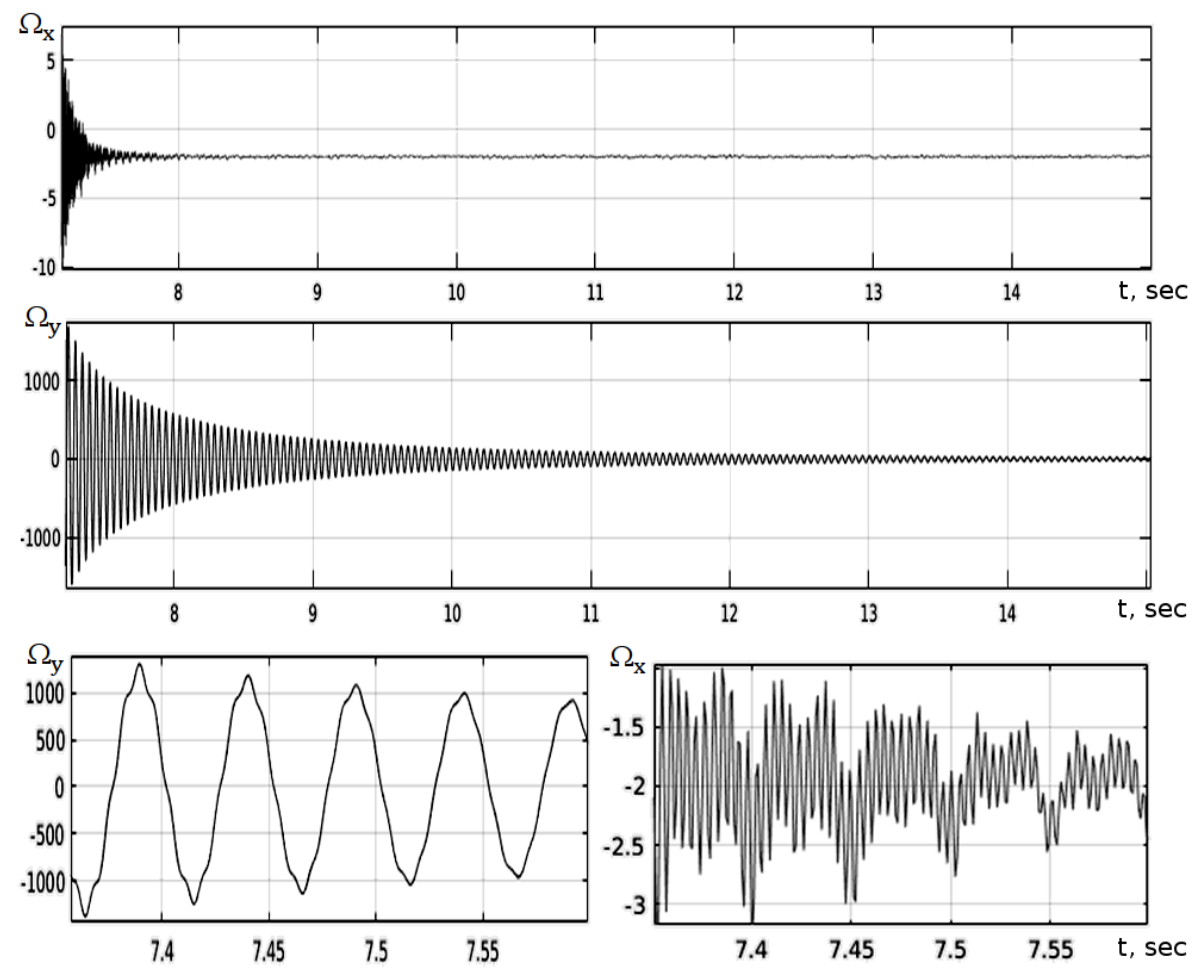

Figure 2. Recording of a cantilever beam vibrations. Angular velocities $\Omega_{x}, \Omega_{y}$. Beam parameters: $b=30 \mathrm{~mm}, L=180 \mathrm{~mm}$

\section{Calculation of the aerodynamic forces acting on the beam from the experimental data}

Let us consider the flexural oscillations of the beam according to the fundamental mode. Under the influence of aerodynamic forces and internal damping, the amplitude of the oscillations $A(t)$ slowly decayed, and the fundamental frequency $\omega$ weakly varied with time $t$ near the natural frequency $\omega_{0}$.

To calculate the hydrodynamic forces acting on the beam from the gyro data the following algorithm was developed:

1. The values of local maxima of $\Omega_{y}(t) \bar{\Omega}_{y}^{1}, \bar{\Omega}_{y}^{2}, \ldots$ and the corresponding moments of time $t_{1}, t_{2}, \ldots$ are found. 
2. On each interval $\left[t_{k}, t_{k+1}\right]$ a harmonic approximation is found in the form

$$
\Omega_{y}^{A p p r}(t)=\varepsilon_{k}+\Omega_{y}^{k} \cos \left(\omega_{k} t+t_{0 k}\right)
$$

Four required parameters $\left(\varepsilon_{k}, \Omega_{y}^{k}, \omega_{k}, t_{0 k}\right)$ are found from the condition of minimum of the root-mean-square deviation $\Omega_{y}^{A p p r}$ from $\Omega_{y}$ on the interval considered. As an initial approximation to $\left(\varepsilon_{k}, \Omega_{y}^{k}, \omega_{k}, t_{0 k}\right)$ the next values $\left(0,0.5\left(\bar{\Omega}_{y}^{k}+\bar{\Omega}_{y}^{k+1}\right), 2 \pi /\left(t_{k+1}-t_{k}\right), 0\right)$ are chosen.

3. The resulting approximation at each time interval $\left[t_{k}, t_{k+1}\right]$ is used to calculate the amplitude of flexural vibrations of the free end of the beam

$$
A_{k}=\sin \left(\Omega_{y}^{k} / \omega_{k}\right) / \frac{d W}{d x}(L),
$$

where $W$ is the profile of oscillations.

4. The $\operatorname{LD} \delta(t)=-2 \pi \omega_{0}^{-1} A^{-1}(d A / d t)$ and $\operatorname{RVF} \gamma_{P}=\left(\omega_{0}-\omega\right) / \omega_{0}$ of the beam oscillation as a function of time are calculated. Further, for each experiment in the range of the realized oscillation amplitudes the dependences $\delta(A), \gamma(A)$ are built.

5. The dependencies $\delta(A), \gamma(A)$ are used for the calculation of the aerodynamic forces acting on each cross-section of the beam. For this, the formulas obtained in [10] are applied:

$$
\begin{gathered}
C_{D}(\kappa, \beta, \Delta)=\frac{4.61}{\kappa \sqrt{\beta}}+\frac{9}{32} \frac{\rho}{\rho_{a}} \Delta \kappa^{-8 / 3} \frac{d}{d \kappa}\left(\kappa^{8 / 3}(\delta(\kappa)-\delta(0))\right), \\
C_{M}(\kappa, \beta, \Delta)=\frac{3}{\pi} \frac{\rho}{\rho_{a}} \Delta \kappa^{5 / 3} \frac{d}{d \kappa}\left(\kappa^{8 / 3} \gamma(\kappa)\right) .
\end{gathered}
$$

Here $C_{D}, C_{M}$ are the local coefficients of aerodynamic drag and added mass of the beam, $\kappa=A / b$ is the dimensionless amplitude of oscillations, $\beta=b^{2} \omega /(2 \pi v)$ is the dimensionless frequency, $\Delta=h / b$ is the dimensionless beam thickness, $v$ is the kinematic viscosity of the air, $\rho_{a}$ and $\rho$ are the air density and mass density of the beam, respectively.

\section{Verification of results}

The results of the measurements of the aerodynamic forces acting on oscillating beams obtained by the presented method are shown in Figures 3- 4. The changes of $C_{D}$ and $C_{M}$ are presented in dependence on two control parameters: the dimensionless frequency and the dimensionless amplitude of oscillations. The variation of the third parameter $\Delta$ in the considered range $[1 / 30,1 / 20]$ did not have a significant effect on the measurement results.

As can be seen, the influence of the dimensionless frequency on the drag coefficient (see Figure 3 ) is observed only for small oscillation amplitudes. At large amplitudes $(\kappa>0.8)$ $C_{D}$ functions for all $\beta$ values have the same asymptotic behaviour. Comparing results with the known data, let us pay attention to the large range of variation of $C_{D}$ estimates presented in the literature. The results obtained lie at the lower boundary of this range and are in good agreement with the experimental data $[10,13,14]$.

The results of measuring of the added mass coefficient are shown in Figure 4. As can be seen, $C_{M}$ is largely dependent on both parameters of the oscillatory process. The range of $C_{M}$ estimates for thin plates presented in the literature is also quite wide. The data obtained are generally in good agreement with the results $[3,6,10]$. 


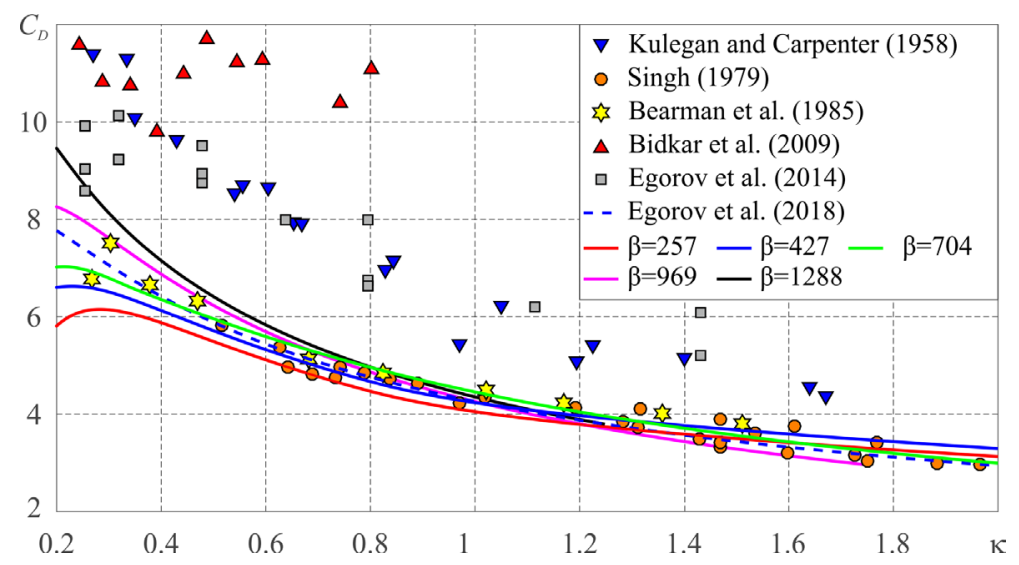

Figure 3. Drag coefficients $C_{D}$ vs dimensionless amplitude $\kappa$ for different values of $\beta$

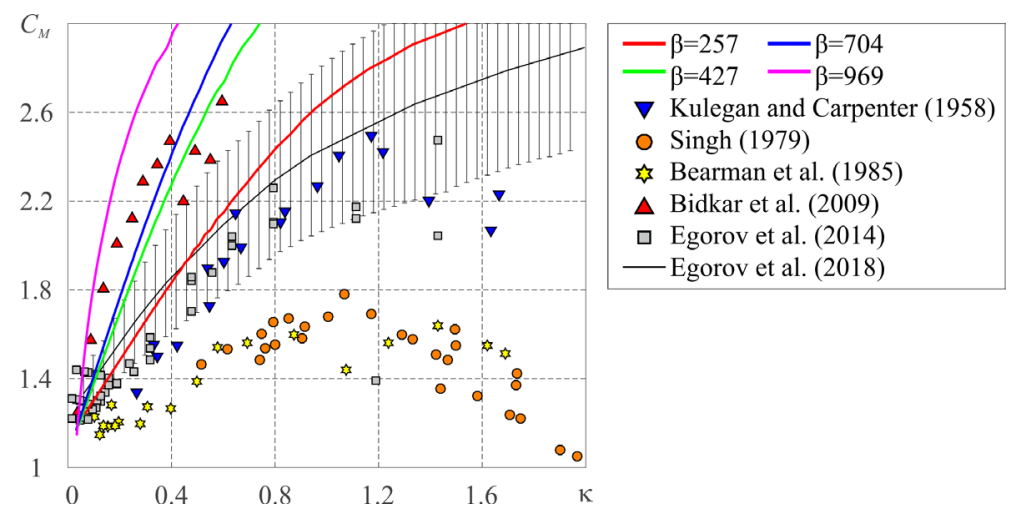

Figure 4. Coefficient of added mass $C_{M}$ vs dimensionless amplitude $\kappa$ for different values of $\beta$

\section{Conclusions}

In the paper we present a method for determining of the aerodynamic influence on oscillating cantilever beams based on gyroscope measurements. To verify the method an extensive series of experiments were carried out. Verification results show a good correspondence of the obtained aerodynamic force estimates with the known numerical and experimental data practically in the entire range of the investigated parameters.

This work was funded by the subsidy allocated to Kazan Federal University for the state assignment in the sphere of scientific activities 9.9786.2017/8.9. 


\section{References}

[1] J. E. Sader, Journal of Applied Physics 84, 64-76 (1998)

[2] M. Kimber, R. Lonergan, S.V. Garimella, Journal of Fluids and Structures 25, 1334-1347 (2009)

[3] R. Bidkar, M. Kimber, A. Raman, A. Bajaj, S.V. Garimella, Journal of Fluid Mechanics 634, 269-289 (2009)

[4] L. Tao, K. Thiagarajan, Applied Ocean Research 25, 53-62 (2003)

[5] L. Tao, K. Thiagarajan, Applied Ocean Research 25, 21-35 (2003)

[6] A.G. Egorov, A.M. Kamalutdinov, A.N. Nuriev, V.N. Paimushin, Mechanics of Composite Materials 50, 267-278 (2014)

[7] M. Aureli, M. Porfiri, Applied Physics Letters 96, 164102 (2010)

[8] M. Aureli, M. Porfiri, M.E. Basaran, Journal of Sound and Vibration 331, 1624-1654 (2012)

[9] C.N. Phan, M. Aureli, M. Porfiri, Journal of Fluids and Structures 40, 52-69 (2013)

[10] A.G. Egorov, A.M. Kamalutdinov, A.N. Nuriev, Journal of Sound and Vibration 421, 334-347 (2018)

[11] B.Shrestha, S.N. Ahsan, M. Aureli, Physics of Fluids 30, 013102 (2018)

[12] G.H. Keulegan, L.H. Carpenter, Journal of Research of the National Bureau of Standards 360, 423-440 (1958)

[13] S. Singh, Forces on Bodies in Oscillatory Flow (Ph.D. thesis, University of London, 1979).

[14] P.W. Bearman, M.J. Downie, J.M.R. Graham, E.D. Obasaju, Journal of Fluid Mechanics 154, 337-356 (1985) 